\title{
Propuesta de gamificación para el aprendizaje de los trastornos del lenguaje: Panel de diagnósticos
}

\author{
Lucía Buil-Legaz ${ }^{a}$, Àngels Esteller-Cano ${ }^{b}$, Eva Aguilar-Mediavilla ${ }^{c}$ y María Martínez- \\ Beceiro $^{\mathbf{d}}$ \\ ${ }^{a}$ Universitat de les Illes Balears (lucia.buil@uib.es), ${ }^{b}$ Universitat de les Illes Balears \\ (angels.esteller@uib.es), 'Universitat de les Illes Balears (eva.aguilar@uib.es), ${ }^{\mathrm{d}}$ Universitat de les Illes \\ Balears (maria.martinez19@estudiant.uib.cat)
}

\begin{abstract}
The purpose of this project is to introduce gamification techniques in the classroom with the aim of motivating students to learn complex content, such as language disorders in school age, in the subject Psychoeducational intervention in language difficulties in the school context, of the second year of the Degree in Primary Education. Thus, we intend to work from a meaningful and experiential learning through a "contest" in which the participants start from a case and have to collect clues to reach the diagnosis. From this methodology, it is intended to produce a connection between the student and the theoretical content, improve their skills, autonomy and promote the relationship between students to improve the classroom environment.
\end{abstract}

Keywords: gamification, active methodology, educational innovation, university.

\begin{abstract}
Resumen
La finalidad de este proyecto es introducir técnicas de gamificación en el aula con el objetivo de motivar a los/las estudiantes hacia el aprendizaje de contenidos complejos, como son los trastornos del lenguaje en edad escolar, en la asignatura Intervención psicoeducativa en las dificultades del lenguaje en el contexto escolar, de segundo curso del Grado de Educación Primaria. Así, se pretende trabajar a partir de un aprendizaje significativo y vivencial a través de un "concurso" en el que los/las participantes parten de un caso y tienen que ir recabando pistas para llegar al diagnóstico. A partir de esta metodología se pretende producir una conexión entre el/la estudiante y el contenido teórico, mejorar sus competencias, la autonomía y promover la relación entre el alumnado para mejorar el ambiente del aula.
\end{abstract}

Palabras clave: gamificación, metodología activa, innovación educativa, universidad.

Financiación: este trabajo está asociado al proyecto de innovación educativa y mejora de la calidad docente PID 181906 (Institut de Recerca i Innovació Educativa, Universitat de les Illes Balears). 


\section{Introducción}

El cambio de paradigma educativo que trajo consigo el Espacio Europeo de Educación Superior (EEES) sitúa al profesor/a como pieza clave en dotar al alumnado de herramientas y competencias suficientes para adquirir diversos contenidos. Así, el/la docente es responsable de brindar al alumnado habilidades suficientes para lograr que realice un trabajo autónomo y continuado, que tiene como punto de partida la motivación hacia el aprendizaje.

Para motivar al alumnado es necesaria la innovación educativa, pero sin dejar de contemplar el trasfondo pedagógico de las actividades que se plantean. Una de las propuestas que se está llevando a cabo cada vez más en los últimos años es la gamificación, o la introducción del juego como herramienta pedagógica. Diversos autores destacan algunos puntos clave en la definición de este proceso, como el propósito que persigue la gamificación al hacer que un producto, servicio o aplicación sea más divertido, atractivo y motivador (Deterding, 2011), lograr la participación e implicación del usuario/a o conseguir involucrar a los usuarios/as (Zichermann, 2012). Este tipo de actividades se han empezado a implementar ya en contextos universitarios existiendo diversos precedentes como las descritas en Contraras y Euguia (2016),

Después de varios años aplicando mejoras metodológicas en nuestra asignatura de segundo curso del Grado de Educación Primaria (Intervención psicoeducativa en las dificultades del lenguaje en el contexto escolar) de la Universitat de les Illes Balears nos dimos cuenta de que uno de los contenidos teóricos que más dificultad presentaba para el alumnado era el tema de los trastornos del lenguaje oral en la edad escolar. En los primeros años de la asignatura asignábamos a cada grupo de prácticas un trastorno de entre una lista de ocho posibles para que preparasen una presentación oral. Esta metodología era útil para que profundizaran en uno de ellos, pero cada vez éramos más conscientes de que no llegaban a adquirir los conocimientos suficientes de aquellos trastornos que no les habían sido asignados. Por esta razón, decidimos introducir algunas modificaciones en el trabajo práctico que acompaña a este apartado de la teoría. La innovación consistió en introducir un juego tipo concurso en el cual el alumnado parte de la descripción de un caso para, por grupos, ir pidiendo pistas y desvelando más información con el fin de llegar a averiguar el diagnóstico del caso presentado. De esta forma, como señalan Foncubierta y Rodríguez (2015), a partir de la incorporación de juegos solucionamos problemas como la dispersión, la inactividad, la no comprensión o la sensación de dificultad mediante el acto de involucrar al alumnado, además de conseguir una mayor implicación en la actividad. Así, con este cambio de metodología, destacamos una de las grandes ventajas de la gamificación en la medida que trabajan los contenidos a partir de experiencias, lo que proporciona a los/las alumnos/as una mayor sensación de control de los aprendizajes y maximiza la autonomía (Hamari y Koivisto, 2013).

Además, el juego previsto iba a incluir el aprendizaje por resolución de problemas, el aprendizaje cooperativo y un pequeño componente competitivo que no afectaba a la nota, aunque sí a la consecución de un regalo simbólico (Cantador, 2016). Aunque existe un cierto debate de si es adecuado el uso de la competición en educación (Thousand, Villa y Nevin, 1994), los resultados de estudios previos muestran el efecto beneficioso y motivador de una competición siempre que el premio sea simbólico, la competición breve y esta se vea como 
un proceso y no como el resultado (Cantador, y Conde, 2010). Por otro lado, el aprendizaje por resolución permite simular una situación profesional en la que el alumno debe tomar decisiones como las que se encontraría en ella (Schmidt, 1983; Schmidt, Rotgans, y Yew, 2011). Finalmente, el aprendizaje cooperativo, en el que cada alumno tiene un trabajo asignado pero la compleción una tarea depende del trabajo del grupo completo, permite un enriquecimiento del producto final, además del trabajo de competencias en habilidades sociales (Johnson \& Johnson, 1999).

\section{Objetivos}

El objetivo principal del proyecto es desarrollar e implementar una técnica docente que permita:

- Mejorar la comprensión de aspectos teóricos más complejos por parte del alumnado

- Impulsar la motivación por el aprendizaje de los trastornos del lenguaje en edad escolar

- Trabajar a través del aprendizaje significativo y vivencial

- Mejorar las competencias y la autonomía hacia el aprendizaje

- Implicar al alumnado en la resolución de tareas

- Generar competitividad como herramienta para promover la colaboración

\section{Desarrollo de la innovación}

El proyecto llevado a cabo ha consistido en la transformación de una práctica que anteriormente estaba planteada desde un formato tradicional. En esta tarea práctica un grupo de alumnos/as tenían que hacer la presentación oral de un tema y el resto simplemente actuaban de oyentes. La transformación de la tarea práctica gira hacia una actividad más dinámica, participativa y significativa para el alumnado en la que todos los grupos participan, interactúan y están interconectados.

Para ello, los/las alumnos/as se sitúan en grupos de trabajo de entre 4 y 7 personas. Cada miembro del grupo tiene un tema asignado desde el principio del curso de entre siete temas posibles relativos a diferentes trastornos del lenguaje oral en el contexto escolar (disfonía, disfemia, hipoacusia, trastorno específico del lenguaje, dificultades de lenguaje asociadas a discapacidad intelectual, dificultades de lenguaje asociadas a parálisis cerebral y dificultades de lenguaje asociadas a autismo). Desde el día que eligen el tema hasta el día que hacemos la práctica en el aula transcurre un mes. En este tiempo, los miembros de cada grupo se organizan de forma autónoma para hacer una primera fase (en las primeras dos semanas aproximadamente) de investigación y búsqueda de información de manera individual y una segunda fase (en las dos semanas siguientes) de puesta en común con el resto del grupo de trabajo. Para ello cuentan con unas instrucciones específicas sobre qué tipo de información buscar y dónde conseguirla, además de un texto base de resumen sobre cada uno de los trastornos propuestos. 
El día de la práctica en el aula llevamos a cabo el concurso, que consiste en un panel de diagnósticos que comienza con la descripción breve de un caso hipotético. Tras leer esta primera pantalla del caso pasamos a una segunda pantalla en la que aparecen diferentes pruebas de evaluación posibles con los resultados de cada una de ellas oculto. Cada grupo pide su turno para pedir "pistas" a través de unos pulsadores adquiridos específicamente para esta actividad. Cada pista consiste en destapar una de estas pruebas de evaluación y revelar la información que contiene. Por ejemplo, una de las pistas que pueden pedir en un caso es "Pruebas cognitivas". Al destapar la tarjeta, puede aparecer la siguiente información: CI 65; sin incidencias; CI dentro de los rangos de la normalidad; discrepancia entre CI verbal y manipulativo...

A partir de la recopilación de pistas, junto con la información facilitada en la descripción del caso, cada grupo de trabajo puede llegar a resolver el diagnóstico, que se contaría como correcto únicamente si la justificación del mismo es adecuada.

El grupo que resuelva de forma correcta cada caso irá sumando puntos, mientras que los diagnósticos incorrectos restan puntos. El equipo que consiga más puntos al final de la actividad tendrá una recompensa material, algo simbólico que conocerán de antemano, como un llavero realizado por usuarios del programa de terapia ocupacional de ISLA, organización Mater Misericordiae. Por otra parte, las docentes contamos con una plantilla de evaluación para poder hacer un registro sobre la adquisición de contenidos a partir de las intervenciones de cada grupo. Esta plantilla de evaluación se utilizará para poder otorgar una puntuación numérica en esta actividad práctica que corresponde al 10\% de la nota total de la asignatura.

El desarrollo de este proyecto incluyó diferentes fases, comenzando por una etapa previa de preparación por parte de las docentes de la asignatura para sistematizar la actividad y orientarla de forma que mejore la autonomía del alumnado. Teniendo en cuenta el elevado número de alumnos/as, se planteó la actividad en todos los grupos reducidos de práctica de la asignatura para favorecer una mejor supervisión del proceso. Por último, para llevar a cabo el seguimiento del proceso y evaluación con el fin de recoger las impresiones del alumnado sobre el proyecto se diseñó, por un lado, un cuestionario de satisfacción y, por otro lado, un cuestionario tipo test de aprovechamiento de la actividad. Este cuestionario de aprovechamiento ya se aplicaba antes de la innovación de la misma manera y consistía en 15 preguntas de tipo test sobre los contenidos.

Dado que los grupos reducidos de prácticas están asignados a cada una de las docentes de la asignatura, cada una de nosotras llevó a cabo la dinámica con sus grupos prácticos, si bien la preparación, supervisión y evaluación de la actividad se gestionó por parte de las dos docentes de la asignatura junto con la inestimable ayuda de la alumna colaboradora otorgada por el proyecto.

En total, 220 alumnos y alumnas, dos docentes y una alumna colaboradora participaron en este proyecto. 


\section{Resultados}

Para llevar a cabo la evaluación sobre la percepción de satisfacción por parte del alumnado con la implementación de esta nueva metodología elaboramos un cuestionario sencillo que se facilitó a los/las alumnas a través de un formulario de Google. Todos los ítems presentados se contestaban con una escala de puntuación de 0 a 10. Los ítems utilizados para la evaluación se detallan en la Tabla 1.

Tabla 1. Cuestionario de evaluación de la actividad de gamificación

\begin{tabular}{|l|}
\hline 1. La organización de la práctica en cuanto a tiempo y espacio físico ha sido adecuada. \\
\hline 2. La actividad me ha permitido mejorar la comprensión de aspectos teóricos complejos \\
\hline $\begin{array}{l}\text { 3. Me he sentido motivado/a hacia el aprendizaje de los trastornos del lenguaje oral en } \\
\text { la etapa escolar. }\end{array}$ \\
\hline $\begin{array}{l}\text { 4. Creo que esta metodología permite un aprendizaje significativo sobre contenidos } \\
\text { teóricos. }\end{array}$ \\
\hline 5. He podido conseguir objetivos trabajando de forma autónoma. \\
\hline $\begin{array}{l}\text { 6. Creo que he podido poner en práctica habilidades que no utilizamos en las sesiones } \\
\text { habituales. }\end{array}$ \\
\hline 7. Esta actividad me aporta competencias que puedo utilizar en otras materias. \\
\hline 8. En general estoy satisfecho/a con el desarrollo del proyecto. \\
\hline 9. Recomendaría esta metodología (concurso) como herramienta de aprendizaje. \\
\hline
\end{tabular}

Todos los ítems evaluados recibieron puntuaciones positivas. Las puntuaciones más altas se recogieron en los ítems 2, 4 y 9 con una puntuación promedio de 8,09; 8,06 y 8,35, respectivamente. En cambio, las puntuaciones más bajas se reflejaron en los ítems 5 y 7 , con una puntuación promedio de 7,56 y 7,59, respectivamente.

En cuanto al ítem sobre la satisfacción global con la actividad, la puntuación promedio obtenida fue 7,94 .

Respecto al aprendizaje del contenido a partir de esta actividad, se compararon las notas obtenidas en el cuestionario final de aprendizaje del curso. Se compararon las calificaciones promedio obtenidas en el curso anterior, con la metodología tradicional y las obtenidas al incorporar la nueva metodología. Se encontraron diferencias significativas entre la media de ambos cursos, siendo las medias de las calificaciones superiores tras la innovación docente ( $\mathrm{X}$ antes de innovación $=6,3 \mathrm{SD}=2,4 ; \mathrm{X}$ después de innovación $=6,96, \mathrm{SD}=2,26 ; \mathrm{t}$ (300) $=-2.199, \mathrm{p}=.029)$. 


\section{Conclusiones}

Los resultados obtenidos parecen indicar que la propuesta de gamificación, como metodología para el aprendizaje de los trastornos de lenguaje oral en la etapa escolar, es útil como herramienta para fomentar el aprendizaje de conceptos y resulta una actividad de interés para el alumnado. Por otra parte, los ítems relacionados con la autonomía y la transferencia a otras materias fueron los reportados como mayor impedimento para recomendar la actividad, a pesar de que las puntuaciones no eran bajas.

Teniendo en cuenta que los principales objetivos de esta propuesta eran mejorar la comprensión de aspectos teóricos más complejos por parte del alumnado e impulsar la motivación al trabajar a partir de un aprendizaje activo y vivencial, los resultados muestran que esta actividad parece ser un buen punto de partida para conseguirlos y a partir de aquí impulsar más actividades que sigan la misma línea.

La valoración en conjunto de la propuesta de actividad para la introducción de la gamificación en el aula como herramienta de aprendizaje es positiva tanto para el equipo docente como para el alumnado. En los próximos cursos incorporaremos las modificaciones y adaptaciones que sean necesarias para conseguir mejores resultados en aquellos ítems que hayan obtenido puntuaciones más bajas.

En el futuro, trataremos de aplicar las modificaciones necesarias, así como las sugerencias recogidas de los alumnos/as para hacer de esta actividad una metodología que otros compañeros/as puedan aplicar en sus asignaturas.

\section{Referencias}

CONTRERAS, R., y EUGUIA, J. L. (2016). Gamificación en aulas universitarias. Bellaterra (Cerdanyola del Vallès): Institut de Comunicació Universitat Autònoma de Barcelona. https://doi.org/10.1007/s13398-014-0173-7.2

CANTADOR, I. (2016). "La competición como mecánica de gamificación en el aula: una experiencia aplicando aprendizaje basado en problemas y aprendizaje cooperativo por iván cantador". En: Contreras, R., y Euguia, J. L. (eds.). Gamificación en aulas universitarias. Bellaterra (Cerdanyola del Vallès): Institut de Comunicació Universitat Autònoma de Barcelona. https://doi.org/10.1007/s13398-014-0173-7.2

CANTADOR, I., y CONDE, J. M. (2010). "Effects of competition in education: A case study in an e-learning environment". En: IADIS International Conference e-Learning (pp. 11-18). Freiburg, Alemania: IADIS International Association for Development of the Information Society.

DETERDING, S. (2011). "Meaningful play: Getting gamification right”, Google Tech Talk.

FONCUBIERTA, J. y RODRÍGUEZ, C. (2015). Didáctica de la Gamificación en la clase de español. Editorial Edinumen. $<$ https://www.edinumen.es/spanish_challenge/gamificacion_didactica.pdf $>$ [Consulta: 18 marzo de 2019] 
HAMARI, J. y KOIVISTO, J. (2013) "Social motivations to use gamification: an empirical study of gamifying exercise". En: ECIS 2013 Completed Research. 105.

JOHNSON, D., y JOHNSON, R. (1999). Learning Together and Alone: Cooperative, Competitive, and Individualistic Learning. Allyn and Bacon Press

SCHMIDT, H. G. (1983). Problem-based Learning Rationale and Description. Medical Education, 17, 11-16

SCHMIDT, G., ROTGANS, J., \& YEW, E. (2011). The Process of Problem-based Learning: What Works and Why. Medical Education, 45(8), 792-806.

THOUSAND, J., VILLA, A., y NEVIN, A. (1994). Creativity and Collaborative Learning. Brookes Press.

ZICHERMANN, G. "Gamification: Innovation and the Future. 2012." Youtube $<$ https://www.youtube.com/watch?v=kJ4yJjGn2js $>$ [Consulta: 5 de marzo de 2019] 